\title{
The immune system and microorganisms: a love-hate relationship revisited
}

Accepted: 3 January 2003 / Published online: 5 February 2003 (C) Springer-Verlag and SEM 2003

The study of interactions between microorganisms and higher cells or, more generally, between microbiology and immunology, originated with the beginning of these scientific fields. In fact, the development of immunology as a scientific discipline was a logical consequence of the germ theory of disease. In the mid-nineteenth century, remarkable contributions were made by the French and German schools, which approached immunology and bacteriology as a single scientific discipline, and which had in Pasteur and Koch, respectively, their most outstanding representatives. We must also remember that it was the confrontation between these two schools that led to the splitting of immunology into two branches-humoral and cell-mediated-, a separation that is still maintained.

Due to the increasing amount of knowledge and the rapid advancements in microbiology and immunology, as well as in microbial ecology, it has become widely accepted that, during evolution, the cells of multicellular organisms have established essential relationships with many prokaryotic and lower eukaryotic organisms, with which they coexist. This suggests an exquisite balance between the elimination of microorganisms by the immune system and the spread and growth of colonizing microorganisms. Nevertheless, there are many other organisms - prokaryotes, eukaryotes, and viruses - against which multicellular organisms orchestrate a defensive response aimed at their elimination from areas that must be kept aseptic. Regarding pathogenic microorganisms, there must be also a balance between colonization and keeping the host alive, since the host's death would imply the pathogen's death too.

\footnotetext{
J. Jiménez $(\bowtie) \cdot M$. Sánchez-Pérez

Departamento de Microbiogía y Genética,

Instituto de Microbiología Bioquímica,

Universidad de Salamanca/CSIC,

Av. Doctores de la Reina s/n, 37007 Salamanca, Spain

E-mail: javierj@usal.es

Tel.: + 34-923294400

Fax: + 34-923224876
}

The incidence of infectious diseases has greatly increased over the last two decades. One reason for this is the renewed increase in the occurrence of microorganisms responsible for diseases, such as tuberculosis, which in the past had been nearly eliminated by antibiotics. In addition, there is a higher incidence of infections subsequent to transitory immunodeficiencies such as those caused by extremely invasive medical procedures, including anticancer therapies, the general use of broadspectrum antibiotics, the enormous development of organ transplantation, and the emergence of virus-induced diseases that cripple the immune system, e.g. AIDS. Consequently, some microorganisms that are usually harmless commensals have the potential of becoming opportunistic pathogens; this is the case of Candida albicans, which is responsible for both surface and systemic infections. The occurrence of other microorganisms, including the filamentous fungus Aspergillus fumigatus, which causes hemorrhagic fever, has also drastically increased. Finally, external phenomena including climatic change, the invasion by humans of new geographical areas and niches, and human migration all present new challenges for the human immune system.

Efforts to help the immune system to overcome the challenges posed by "modern times" have mainly been focused along two lines of research: (1) an intensive screening for new drugs to more effectively control emerging infectious diseases; and (2) attempts to identify the mechanisms by which the immune system controls the occurrence of pathogenic microorganisms. The study of cellular targets for new antimicrobial agents has led to the production of very selective drugs with fewer side effects on host cells. New anti-AIDS drugs allow AIDS patients to have acceptable life expectancies and quality of life, at least in developed countries. Three types of clinical trials of an AIDS vaccine have been carried out in Thailand and the USA; the results should soon be available. However, assays with monkeys vaccinated against AIDS have shown that the vaccines are palliative rather than protective, decelerating the progression of AIDS but not conferring immunity against the disease. 
Vaccinated monkeys become infected but AIDS proceeds at a slower pace. New antifungal agents - now in the last steps of clinical trials - disrupt structures of fungal cell walls. Great efforts have also been made to design new methods and protocols that allow early diagnosis of these infections.

Regarding the mechanisms employed by the immune system to fight different kinds of infections, much has been learned about the modulation of the TH1/TH2 response by helper-T-lymphocytes. It is the outcome of this response that determines the susceptibility or resistance of the host to a given microorganism. The studies carried out in C. albicans provide an example. C. albicans exhibits a dimorphic transition from yeast to a mycelium, the latter being the infectious agent. It has been increasingly accepted that as a single-celled yeast C. albicans induces a TH1 (cell-mediated) response that efficiently controls if not eliminates the infection. When C. albicans grows as a mycelium, however, it "hides" from the TH1 protective response and induces a TH2 (humoral) response which is not efficient against C. albicans infection. The C. albicans model exemplifies how an understanding of immune system processes can help us to modulate them externally by using the appropriate drugs and thereby lead or assist the immune system in coping with the challenges that "modern times" present.

At this moment, the new fields of genomics and proteomics merit some attention. The sequencing of whole genomes and the study of their protein maps have generated an amazing amount of information not only about microorganisms but also about higher organisms. These areas of research have provided us with powerful tools for better understanding the structure, function, and biology of both microorganisms and immune systems.

We cannot end this discussion without mentioning the efforts also made in vaccine research. Current trends include the use of recombinant vaccines, live viral vectors, conjugated vaccines, DNA vaccines, and anti-tumoral vaccines. Much of this research tries to solve limitations of traditional vaccines, both economical and those related to preservation and distribution, which have prevented their use in some geographical regions. New protocols for vaccine administration, including nasal, vaginal, and skin patches, along with new strategies for vaccination, offer a promising future. 